\title{
Long-term efficacy of tiotropium in relation to smoking status in the UPLIFT trial
}

\author{
D.P. Tashkin*, B. Celli ${ }^{\#}$, S. Kesten ${ }^{\oplus}$, T. Lystig ${ }^{\star}$, S. Mehra ${ }^{+}$and M. Decramer ${ }^{\S}$
}

ABSTRACT: UPLIFT (Understanding Potential Long-Term Improvements in Function with Tiotropium), a 4-yr trial of tiotropium in chronic obstructive pulmonary disease, allowed for assessment of smoking status on long-term responses to maintenance bronchodilator therapy.

5,993 patients were randomised (tiotropium/placebo). Lung function, St George's Respiratory Questionnaire, exacerbations and adverse events were followed. Patients were characterised as continuing smokers (CS), continuing ex-smokers (CE), or intermittent smokers (IS) based on selfreporting smoking behaviour.

$60 \%, 14 \%$ and $26 \%$ of patients were CE, CS and IS, respectively. The rate of forced expiratory volume in $1 \mathrm{~s}$ (FEV 1$)$ decline for placebo patients was most rapid in CS $(-51 \pm 4,-36 \pm 2$ and $-23 \pm 2 \mathrm{~mL} \cdot \mathrm{yr}^{-1}$ in CS, IS, and CE, respectively). Tiotropium did not alter FEV 1 decline, but was associated with significant improvements in pre- and post-bronchodilator FEV1 over placebo that persisted throughout the 4-yr trial for each smoking status (pre-bronchodilator: 125, 55 and $97 \mathrm{~mL}$ at 48 months in CS, IS and CE, respectively; $p \leqslant 0.0003)$. Tiotropium reduced exacerbation risk in CS (HR (95\% Cl) $0.81(0.68-0.97))$, in CE (0.86 (0.79-0.93)) and trended towards significance in IS (0.89 (0.80-1.01)). At 4 yrs, St George's Respiratory Questionnaire for tiotropium patients improved the most in CS $(-4.62$ units, $p=0.0006)$ and the least in IS $(-0.54$ units, $p=0.55)$, compared with control.

Tiotropium provided long-term benefits irrespective of smoking status, although differences among categories were observed.

KEYWORDS: Chronic obstructive pulmonary disease, forced expiratory volume in $1 \mathrm{~s}$ rate of decline, smoking behaviour, tiotropium

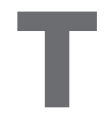

he relationship between smoking behaviour and long-term responses to maintenance bronchodilator therapy has not been thoroughly evaluated. When comparing the efficacy of the long-acting anticholinergic bronchodilator, tiotropium, in a 3-month study between 80 smokers and 224 ex-smokers with chronic obstructive pulmonary disease (COPD), MoITA et al. [1] previously reported twice as large a placeboadjusted improvement in pre-bronchodilator forced expired volume in $1 \mathrm{~s}$ (FEV1) in the smokers $(138 \mathrm{~mL})$ than the ex-smokers $(66 \mathrm{~mL})$, although the difference in the response to tiotropium between the two smoking groups was not statistically significant. In contrast, in a pooled analysis, stratified by smoking status, of seven clinical trials in which the short-acting anticholinergic bronchodilator, ipratropium, was compared with a $\beta_{2^{-}}$ agonist over a 90-day treatment period in a total of 1,836 subjects with moderately severe COPD, the improvement in baseline lung function in the ipratropium-treated patients was found to be more marked in ex-smokers than current smokers [2].

The 4-yr multinational placebo-controlled trial of tiotropium versus placebo in 5,993 subjects with COPD (UPLIFT, Understanding Potential LongTerm Improvements in Function with Tiotropium) [3] provided an opportunity to assess more fully the potential relationship between smoking status and both lung function and patient-reported outcomes of maintenance therapy with a long-acting muscarinic antagonist over an extended period of time in a large group of continuing smokers, intermittent smokers and ex-smokers with COPD, taking into account the potentially confounding influence of concomitant respiratory medications.

\section{METHODS}

\section{Study design}

UPLIFT was a 4-yr, randomised, placebo-controlled clinical trial in 5,993 patients with COPD. The
AFFILIATIONS

*David Geffen School of Medicine UCLA, Los Angeles, CA,

${ }^{\#}$ Caritas St. Elizabeth's Medical Center, Boston, MA,

"Boehringer Ingelheim

Pharmaceuticals, Ridgefield, CT

+Pfizer Pharmaceuticals, New York, NY, USA.

§University of Leuven, Leuven, Belgium.

CORRESPONDENCE

D.P. Tashkin

Dept of Medicine

David Geffen School of Medicine UCLA

10833 Le Conte Avenue

Los Angeles

CA 90095-1690

USA

E-mail: DTashkin@mednet.ucla.edu

Received:

May 262009

Accepted after revision:

Aug 142009

First published online:

Aug 282009 
methods have been previously published in detail as have the main results $[3,4]$. In brief, key inclusion criteria consisted of the following: $\geqslant 40$ yrs of age, smoking history of at least 10 packyrs, post-bronchodilator FEV1 to forced vital capacity (FVC) ratio of $\leqslant 0.70$ of predicted, and post-bronchodilator FEV1 of $\leqslant 70 \%$ predicted. Key exclusion criteria included: COPD exacerbation within 4 weeks prior to screening, respiratory infection within 4 weeks prior to screening, history of asthma, prior pulmonary resection, and use of supplemental oxygen for $>12 \mathrm{~h} \cdot \mathrm{day}^{-1}$. The protocol was approved by the local ethics committees and all patients provided written informed consent.

Patients were randomised to receive either tiotropium or placebo. All patients were permitted to use other maintenance respiratory medications throughout the trial with the exception of inhaled anticholinergics. The co-primary end-points were the annual rate of change of both pre- and post-bronchodilator FEV1 from 30 days after randomisation through 48 months.

A smoking cessation programme was offered to all patients after consent and prior to randomisation. Of 1,825 patients who were smoking at the time of screening, 150 reported no smoking at baseline, possibly as a consequence of the smoking cessation programme that was offered to all eligible participants prior to randomisation. Of 4,167 patients who reported abstinence from smoking at screening, 97 reported having relapsed to smoking at randomisation. For the analysis described herein, patients were also grouped according to on-trial smoking status, i.e. as to whether they were continuing smokers, continuing ex-smokers, or intermittent smokers during the course of the study. Continuing smokers consisted of patients who were recorded as smoking at baseline and having continued to smoke at all clinic visits. Continuing exsmokers consisted of patients who were recorded as having quit smoking prior to randomisation and having maintained smoking abstinence at all clinic visits. Intermittent smokers were defined as subjects who changed their smoking behaviour from randomisation on at least one clinic visit.

\section{Statistical analysis}

The decline of pulmonary function over time was analysed with random coefficient regression in which the FEV1 changed linearly after 30 days for each patient. Individual intercepts and slopes were random following bivariate normal distributions with different means for each treatment group, and a common covariance matrix. The same model was used for St George's Respiratory Questionnaire (SGRQ) decline over time (from 6 months until completion of the study). All patients who underwent randomisation and received study drug and who had at least three post-randomisation measurements of pulmonary function (at least two for SGRQ) were used in the analyses of decline. SGRQ values from Turkey were excluded due to an error in the translation of the questionnaire.

The values of pulmonary function tests at specific time-points throughout the study were modelled using mixed model repeated measures analysis of covariance with an unstructured covariance matrix. The same restriction to subjects with three post-randomisation measurements of pulmonary function (two for SGRQ) as in the analyses of decline was used.

Cox regression was used for the time to event end-points of exacerbations and mortality. For exacerbations and exacerbations leading to hospitalisation, the number of events and event days were compared between the study groups with relative risks through the use of Poisson regression with correction for overdispersion.

Analyses were performed with SAS software, version 8.2 (SAS Institute, Cary, NC, USA). All reported p-values are two-sided and not corrected for multiple testing. Details of the statistical analysis plan are described in the report by TASHKIN et al. [3].

\section{RESULTS}

\section{Patient demographics}

A total of 5,992 randomised COPD patients were included in the analysis (fig. 1). With respect to on-trial smoking status, information is available on a subset of 5,925 patients. At baseline, $70 \%$ of the patients were ex-smokers and $30 \%$ were active smokers. Over the course of the trial, $60 \%$ of the patients were continuing ex-smokers, $14 \%$ continuing smokers and $26 \%$ were intermittent smokers (table 1). Of the ex-smokers at baseline, $85 \%$ in the placebo group and $83 \%$ in the tiotropium group remained ex-smokers, while $14 \%$ in the placebo group and $16 \%$ in the tiotropium group had at least one clinic visit in which they reported smoking. Of the patients who were active smokers at baseline, $48 \%$ in the placebo group and $47 \%$ in the tiotropium group remained smokers, while 50\% in the placebo group and $52 \%$ in the tiotropium group had at least one clinic visit in which they reported not smoking. A greater proportion of females than males were intermittent smokers. Mean age varied between 61 and 66 yrs for the different smoking categories. Global Initiative for Chronic Obstructive Lung Disease (GOLD) stage II and III patients accounted for between $89 \%$ and $93 \%$ of the patient population in each smoking status category.

While for most demographic variables the distribution between tiotropium- and placebo-treated patients by smoking category was generally similar, notable disparities existed in relation to both sex and baseline disease severity (table 1). A greater percentage of continuing smokers randomised to receive tiotropium were male $(72 \%)$ than the percentage of continuing smokers randomised to receive placebo ( $62 \%$ male). Continuing ex-smokers differed in the percentage of individuals in GOLD stages II and III by randomisation group. For continuing ex-smokers randomised to placebo, $41 \%$ and $48 \%$ were in GOLD stages II and III at baseline, respectively. For

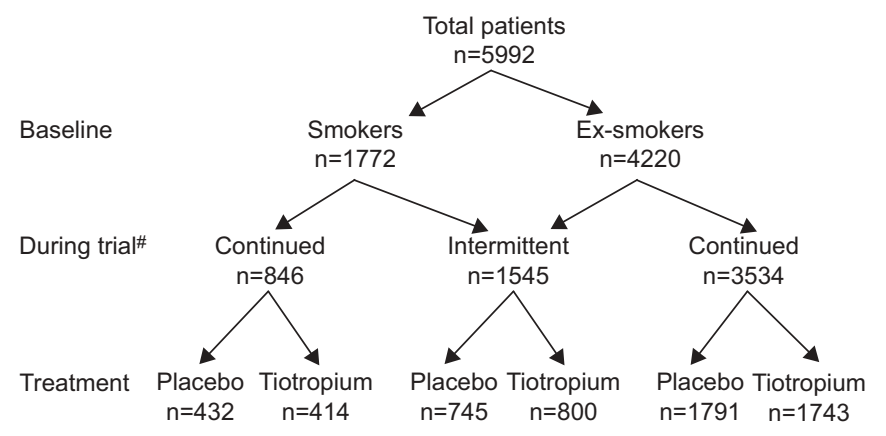

FIGURE 1. Patient numbers according to smoking status and treatment allocation. ${ }^{*}$ smoking status known during trial, $\mathrm{n}=5925$. 
TABLE 1 Baseline demographics of continuing smokers, intermittent smokers and continuing ex-smokers

\begin{tabular}{|c|c|c|c|c|c|c|c|c|c|}
\hline & \multicolumn{3}{|c|}{ Continuing smokers } & \multicolumn{3}{|c|}{ Intermittent smokers } & \multicolumn{3}{|c|}{ Continuing ex-smokers } \\
\hline & Overall & Tiotropium & Placebo & Overall & Tiotropium & Placebo & Overall & Tiotropium & Placebo \\
\hline Patients $\mathbf{n}$ & 846 & 414 & 432 & 1545 & 800 & 745 & 3534 & 1743 & 1791 \\
\hline Total patients in trial \% & 14.3 & 7.0 & 7.3 & 26.1 & 13.5 & 12.6 & 59.6 & 29.4 & 30.2 \\
\hline Male \% & 66.5 & 71.5 & 61.8 & 73.3 & 74.9 & 71.5 & 77.1 & 76.4 & 77.7 \\
\hline$\|$ & 52.2 & 50.5 & 53.9 & 48.2 & 46.6 & 49.9 & 43.2 & 45.3 & 41.2 \\
\hline III & 40.4 & 43.0 & 38.0 & 41.6 & 43.0 & 40.0 & 45.8 & 44.1 & 47.5 \\
\hline IV & 6.4 & 5.8 & 6.9 & 8.3 & 8.3 & 8.5 & 9.3 & 9.1 & 9.5 \\
\hline \multicolumn{10}{|l|}{ Pre-bronchodilator } \\
\hline FEV 1 L & 1.19 & 1.20 & 1.19 & 1.13 & 1.12 & 1.15 & 1.06 & 1.07 & 1.05 \\
\hline FEV 1 L & 1.42 & 1.43 & 1.42 & 1.37 & 1.36 & 1.37 & 1.28 & 1.29 & 1.27 \\
\hline FEV $1 \%$ pred & 49.2 & 48.7 & 49.6 & 48.2 & 47.9 & 48.5 & 46.9 & 47.4 & 46.4 \\
\hline FVC L & 3.18 & 3.20 & 3.16 & 3.15 & 3.14 & 3.16 & 3.05 & 3.04 & 3.05 \\
\hline SVC L & 3.22 & 3.25 & 3.18 & 3.24 & 3.25 & 3.24 & 3.19 & 3.19 & 3.18 \\
\hline SGRQ & 48.6 & 48.5 & 48.8 & 45.6 & 44.9 & 46.4 & 45.3 & 45.4 & 45.1 \\
\hline
\end{tabular}

GOLD: Global Inititative for Chronic Obstructive Lung Disease; FEV1: forced expiratory volume in $1 \mathrm{~s}$; \% pred: \% predicted; FVC: forced vital capacity; SVC: slow vital capacity; SGRQ: St George's Respiratory Questionnaire.

continuing ex-smokers randomised to tiotropium, $45 \%$ and $44 \%$ were in GOLD stages II and III at baseline, respectively.

At baseline pre-and post bronchodilator FEV1 was lowest in continuing ex-smokers (table 1). Similarly, baseline pre-and post-bronchodilator FVC and slow vital capacity (SVC) were lowest in continuing ex-smokers. All three categories of smokers showed a similar degree of reversibility (22-24\% improvement in FEV1) following serial administration of four actuations of ipratropium and albuterol. For each treatment group, post-bronchodilator FEV1/FVC was between $42 \%$ and $46 \%$ of predicted. Mean SGRQ total scores at baseline ranged between 44 and 49 across all smoking groups. Continuing smokers had the highest (worst) SGRQ scores.

\section{Lung function outcomes}

Irrespective of treatment assignment, continuing smokers had the most rapid rate of FEV1 decline and the continuing exsmokers the slowest rate of decline with the intermittent smokers exhibiting an intermediate decline. However, for each smoking category the rate of lung function decline (FEV1) showed similar differences between the tiotropium and control arms (table 2 and fig. 2). Although the primary end-point in UPLIFT was rate of change in FEV1 from 30 days through 48 months post-randomisation and was similar between treatment groups within each of the three smoking behaviour categories, significant improvements in lung function (FEV1, FVC and SVC) were observed with tiotropium compared with the control group within each of the three smoking behaviour categories throughout the trial (table 3, figs 2 and 3 and online supplementary fig. 1). The improvements in the continuing smoking group were numerically larger than in either the exsmoking or the intermittent smoking group. This was evident at both one month and 48 months after the initiation of treatment (table 3).

\section{Exacerbations}

The hazard ratios (tiotropium/control) for time to first exacerbation indicated that tiotropium was associated with a reduced risk of an exacerbation by $19 \%$ and $14 \%$ in continuing smokers and ex-smokers, respectively $(p=0.02$ and $p=0.0002$, respectively) and, to a lesser extent, in intermittent smokers $(11 \%, p=0.062$; table 4). Tiotropium also was associated with a tendency towards reduced exacerbation frequency irrespective of smoking status. The results were statistically significant in continuing ex-smokers in whom the reduction was $16 \%$ compared with placebo. Continuing ex-smokers and intermittent smokers were $13 \%$ and $18 \%$ less likely to experience an exacerbation leading to hospitalisation, respectively, when treated with tiotropium ( $\mathrm{p}=0.036$ for both), although there was no difference in continuing smokers (table 4).

\section{Health-related quality of life}

Tiotropium was associated with improved SGRQ scores at both 6 and 48 months in both continuing smokers and continuing ex-smokers, the effect being largest in the continuing smokers (table 5). There appeared to be a consistency in the magnitude of differences among domains within smoking behaviour groups. Intermittent smokers had the smallest differences numerically with no statistical significance at 48 months; however, larger differences were observed at time points prior to 48 months (range for total score: $-1.35--2.17$ ). 


\begin{tabular}{|c|c|c|c|c|c|}
\hline \multirow[t]{2}{*}{ Patient characteristic } & \multicolumn{2}{|c|}{ Tiotropium } & \multicolumn{2}{|c|}{ Control } & \multirow[t]{2}{*}{ p-value } \\
\hline & Subjects $n$ & $\mathrm{FEV} 1$ rate $\mathrm{mL} \cdot \mathrm{yr}^{-1}$ & Subjects $n$ & FEV 1 rate $\mathrm{mL} \cdot \mathrm{yr}^{-1}$ & \\
\hline \multicolumn{6}{|l|}{ Pre-bronchodilator FEV1 } \\
\hline Continuing ex-smoker & 1486 & $-23 \pm 2$ & 1438 & $-23 \pm 2$ & 0.85 \\
\hline \multicolumn{6}{|l|}{ Post-bronchodilator FEV 1} \\
\hline Continuing smoker & 312 & $-59 \pm 4$ & 305 & $-59 \pm 4$ & 0.98 \\
\hline Intermittent smoker & 758 & $-46 \pm 2$ & 673 & $-48 \pm 3$ & 0.57 \\
\hline Continuing ex-smoker & 1484 & $-33 \pm 2$ & 1432 & $-36 \pm 2$ & 0.19 \\
\hline
\end{tabular}

Data are presented as mean \pm SEM, unless otherwise indicated.

\section{Mortality}

Mortality was assessed using three approaches: 1) during the actual treatment period (first dose to last dose +30 days), 2) during the protocol defined treatment period (4 yrs) including collection of vital status information from prematurely discontinued patients, and 3) at the conclusion of a 30-day period after the protocol-defined treatment period (4-yrs +30 days). Vital status collection was complete for $95 \%$ of patients at 4 yrs and only $75 \%$ at the end of the subsequent 30day washout. For each method of assessing mortality, sustained smokers had the highest mortality rate followed by continuing ex-smokers and then intermittent smokers (table 6). The hazard ratios (tiotropium/control) and 95\% confidence intervals, also displayed in table 6 , indicate reductions in mortality in continuing ex-smokers and intermittent smokers related to tiotropium that were statistically significant in the continuing ex-smokers (19\% risk reduction) both on treatment and during the protocol-defined treatment period (vital status 4 -yrs). However, no benefit of tiotropium on mortality was evident in the continuing smokers or the intermittent smokers.

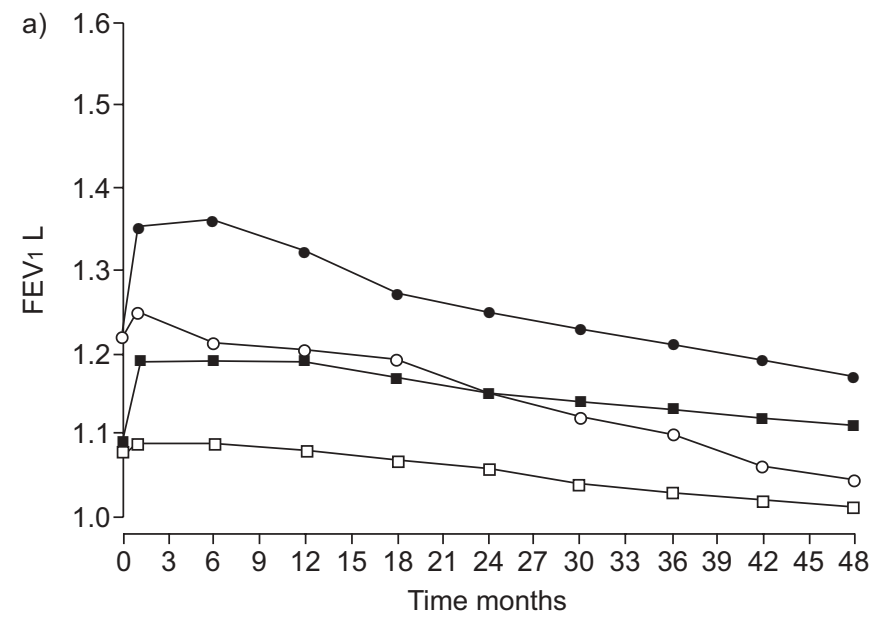

\section{DISCUSSION}

While the efficacy of inhaled corticosteroid therapy has been shown to be impaired by smoking in both COPD and asthma [5-11] and theophylline clearance is increased in smokers, potentially affecting efficacy and toxicity [12], the influence of smoking behaviour on the long-term response to inhaled bronchodilator therapy in COPD has not been well studied. The sparse publications that have addressed this issue are mainly limited to short-term, 3-month studies involving anticholinergic bronchodilators and have yielded somewhat conflicting findings, as described above [1, 2]; one of these studies showed a substantially, although not significantly, greater numerical response to tiotropium in trough FEV1 in continuing smokers than ex-smokers [1], while the other showed a more marked trough FEV1 response to ipratropium in ex-smokers than current smokers [2].

The UPLIFT trial provides a unique opportunity to re-examine this issue in view of its 4-yr duration and the large scope of the trial that included nearly 850 patients who continued to smoke

b)

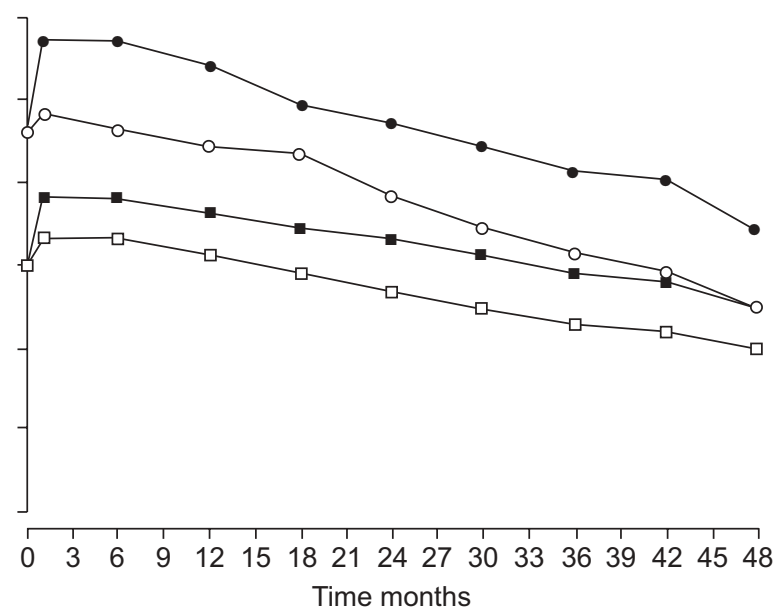

FIGURE 2. a) Pre-bronchodilator forced expiratory volume in $1 \mathrm{~s}$ (FEV1) during 4 yrs. b) Post-bronchodilator FEV1 during 4 yrs. 


\begin{tabular}{|c|c|c|c|c|c|}
\hline \multirow[t]{2}{*}{ Patient characteristic } & \multicolumn{2}{|c|}{ Tiotropium } & \multicolumn{2}{|c|}{ Control } & \multirow[t]{2}{*}{ Difference ${ }^{\star * *}$} \\
\hline & Subjects $\mathrm{n}$ & FEV 1 mL & Subjects $\mathrm{n}$ & FEV $1 \mathrm{~mL}$ & \\
\hline \multicolumn{6}{|l|}{ Pre-bronchodilator FEV1 } \\
\hline Intermittent smokers & 738 & $1130 \pm 10$ & 655 & $1160 \pm 20$ & \\
\hline Continuing ex-smokers & 1448 & $1090 \pm 10$ & 1407 & $1080 \pm 10$ & \\
\hline \multicolumn{6}{|l|}{ Month 1} \\
\hline Continuing smokers & 305 & $1340 \pm 10$ & 298 & $1240 \pm 10$ & 100 \\
\hline Intermittent smokers & 735 & $1240 \pm 10$ & 649 & $1170 \pm 10$ & 70 \\
\hline Continuing ex-smokers & 1036 & $1110 \pm 10$ & 915 & $1010 \pm 10$ & 100 \\
\hline \multicolumn{6}{|l|}{ Post-bronchodilator FEV1 } \\
\hline \multicolumn{6}{|l|}{ Day 1} \\
\hline Continuing smokers & 312 & $1460 \pm 20$ & 303 & $1460 \pm 30$ & \\
\hline Intermittent smokers & 741 & $1360 \pm 20$ & 662 & $1390 \pm 20$ & \\
\hline Continuing ex-smokers & 1463 & $1310 \pm 10$ & 1409 & $1300 \pm 10$ & \\
\hline \multicolumn{6}{|l|}{ Month 1} \\
\hline Continuing smokers & 309 & $1560 \pm 10$ & 302 & $1470 \pm 10$ & 90 \\
\hline Intermittent smokers & 733 & $1440 \pm 10$ & 653 & $1400 \pm 10$ & 40 \\
\hline Continuing ex-smokers & 1457 & $1380 \pm 10$ & 1400 & $1340 \pm 10$ & 40 \\
\hline \multicolumn{6}{|l|}{ Month 48} \\
\hline
\end{tabular}

Data are presented as mean \pm SEM, unless otherwise indicated. ${ }^{*}: p=0.053 .{ }^{* * *}: p<0.001$ for all differences (tiotropium-control), unless otherwise indicated.

throughout the trial and $>3,500$ ex-smokers who maintained abstinence from smoking throughout the entire study period. Moreover, the percentage of patients within these smoking status categories was well balanced at least between the two treatment groups overall, although some imbalance was observed in sex and disease severity: continuing ex-smokers were slightly older, included a higher proportion of males and displayed more severe airflow obstruction than continuing smokers, the intermittent smokers exhibiting intermediate characteristics.

The sub-analysis by smoking status demonstrated that continuing smokers showed a worse outcome than continuing ex-smokers in terms of the rate of decline in both pre- and postbronchodilator FEV1, with intermittent smokers demonstrating intermediate outcomes, irrespective of maintenance anticholinergic therapy, consistent with previous findings from the Lung Health Study $[13,14]$. Conversely, tiotropium was associated with consistently significant improvements in lung function compared to the control arm over the course of the 4-yr study in all smoking behaviour categories, except for a more modest and nonsignificant improvement in post-bronchodilator FEV1 in the intermittent smokers at 4 yrs. Interestingly, consistent with the earlier findings of MOITA et al. [1], the tiotropium-related improvement FEV1 was numerically greater in the continuing smokers than the ex-smokers at trough and even more so when examined after the administration of study drug and four inhalations of albuterol and ipratropium (table 3). However, the results of the study by MoiTA et al. [1] may not necessarily be comparable with the results of the UPLIFT trials due to possible differences in the proportion of subjects using concomitant medications in the two studies. It is tempting to speculate that the apparently greater bronchodilator effect of tiotropium in the continuing smokers may be related to counteraction by the anticholinergic bronchodilator against the well-known bronchoconstrictor effects of cigarette smoke that are believed to be mediated via reflex cholinergic pathways.

Apparent benefits of tiotropium compared with placebo were noted in both the risk for developing an exacerbation and the frequency of exacerbations across all smoking status categories, although these benefits achieved statistical significance only in the continuing ex-smokers. Similarly, tiotropium appeared to be associated with a reduced risk for exacerbations leading to hospitalisation across the three smoking categories, but the difference from placebo was significant only among the continuing ex-smokers and intermittent smokers. Over the 

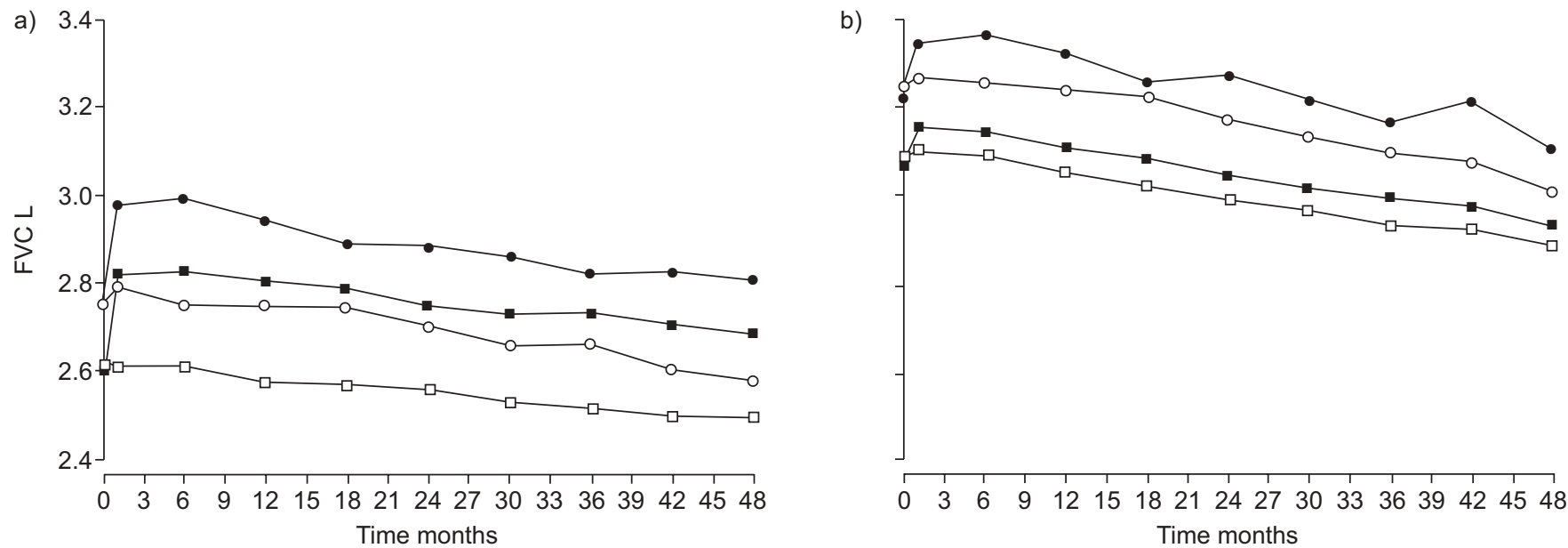

FIGURE 3. a) Pre-bronchodilator forced vital capacity (FVC) during 4 yrs. b) Post-bronchodilator FVC during 4 yrs.

- $\bigcirc$ : continuing smokers.

course of the trial, tiotropium also was associated with improvements in health-related quality of life that were significant in both the continuing smokers and continuing ex-smokers, although the magnitude of the benefit was numerically greatest in the continuing smokers, among whom the benefit exceeded the threshold for a minimal clinically important difference ( $\geqslant 4$ units total SGRQ score). Taken together, these findings suggest a beneficial association of tiotropium with both lung function and patient-reported outcomes in patients with moderate to severe COPD in all subgroups of smoking behaviour, but with different intensity, over the 4-yr course of the study.

Continuing smokers exhibited a higher all-cause mortality rate than subjects in the other smoking categories both ontreatment and over the protocol-defined treatment period, consistent with previous data from the Lung Health Study [13, 14] demonstrating a beneficial effect of smoking cessation and continuing abstinence, as well as of intermittent periods of smoking abstinence, in reducing 14.5-yr mortality, including all-cause mortality and mortality due to coronary heart disease, cardiovascular disease and lung cancer, in subjects with mild-to-moderate COPD [15]. Tiotropium was associated with significantly reduced mortality in the continuing exsmokers while on-treatment and during the 4-yr treatment period with a trend toward a reduction in mortality in the intermittent smokers during the same periods of the study. However, no benefit of tiotropium on all-cause mortality was apparent in the continuing smokers. The latter finding might reflect the higher risk of continuing smokers for fatal cardiovascular events for which a long-acting bronchodilator might not offer sufficient protection.

There are several limitations to the current study. First, notable disparities in baseline characteristics between tiotropium- and placebo-treated patients were observed within the various categories of on-trial smoking behaviour. These imbalances were strongest with respect to sex and baseline disease severity. The possible effects of sex and disease severity on tiotropium effects in UPLIFT are currently under investigation.

\section{TABLE 4 Exacerbation outcomes according to smoking status in the tiotropium and control groups}

\section{Subjects \\ n}

Hazard ratio (tiotropium/control) for exacerbations
Exacerbations

per patient-yr $\mathbf{n}$
Rate ratio

(tiotropium/control)

for number of

exacerbations per

patient-yr

$\begin{array}{lllllll} & 95 \% \mathrm{Cl} & \text { Control } & \text { Tiotropium } & \text { Estimate } & 95 \% \mathrm{Cl}\end{array}$

\section{All exacerbations}

Continuing smokers

Intermittent smokers

Continuing ex-smokers

846

1545

3534

Exacerbation-related hospitalisations

Continuing smokers

Intermittent smokers

Continuing ex-smokers

$\begin{array}{ll}0.80 & 0.67-0.95 \\ 0.89 & 0.79-1.00 \\ 0.85 & 0.79-0.92 \\ & \\ 0.91 & 0.68-1.21 \\ 0.82 & 0.68-0.99 \\ 0.87 & 0.77-0.99\end{array}$

$0.67-0.95$

0.77

0.76

0.83

0.14

0.17

0.16

$0.68-0.99$
$0.77-0.99$
0.87

0.90

0.83

0.99

0.87

0.97
$0.72-1.04$

$0.80-1.01$

$0.77-0.90$

$0.67-1.46$

$0.68-1.11$

$0.82-1.15$ 
TABLE 5 Difference (tiotropium-control) in St George's Respiratory Questionnaire domains at 6 and 48 months

\begin{tabular}{|c|c|c|c|c|c|c|c|c|}
\hline & \multicolumn{4}{|c|}{6 months } & \multicolumn{4}{|c|}{48 months } \\
\hline & Tiotropium & Control & Difference & p-value & Tiotropium & Control & Difference & p-value \\
\hline \multicolumn{9}{|l|}{ Total score } \\
\hline Continuing smokers & 308 & 297 & $-5.43(-7.06--3.80)$ & $<0.0001$ & 207 & 189 & $-4.63(-7.26--2.00)$ & $<0.001$ \\
\hline Intermittent smokers & 717 & 639 & $-2.09(-3.29--0.89)$ & 0.0006 & 543 & 459 & $-0.60(-2.39-1.19)$ & 0.514 \\
\hline Continuing smokers & 308 & 297 & $-5.09(-7.00--3.19)$ & $<0.001$ & 207 & 189 & $-4.15(-7.12--1.17)$ & 0.007 \\
\hline Intermittent smokers & 717 & 639 & $-1.95(-3.33--0.58)$ & 0.0054 & 543 & 459 & $-0.53(-2.49-1.44)$ & 0.6 \\
\hline Continuing ex-smokers & 1428 & 1375 & $-2.17(-3.15--1.19)$ & $<0.0001$ & 1037 & 916 & $-2.40(-3.77--1.03)$ & $<0.001$ \\
\hline \multicolumn{9}{|l|}{ Symptom score } \\
\hline Continuing smokers & 313 & 301 & $-6.02(-8.66--3.38)$ & $<0.0001$ & 210 & 192 & $-4.67(-8.40--0.95)$ & 0.014 \\
\hline Intermittent smokers & 717 & 639 & $-2.24(-3.69--0.80)$ & 0.0024 & 543 & 459 & $-1.49(-3.63-0.66)$ & 0.173 \\
\hline Continuing ex-smokers & 1428 & 1375 & $-2.71(-3.80--1.63)$ & $<0.0001$ & 1037 & 916 & $-3.46(-4.96--1.96)$ & $<0.001$ \\
\hline
\end{tabular}

Data are presented as $\mathrm{n}$ or mean ( $95 \%$ confidence interval), unless otherwise indicated.

Secondly, responses to tiotropium may have been influenced by concomitant therapy with inhaled corticosteroids or theophylline, the potentially confounding effects of which may have been altered by smoking. Thirdly, smoking status was not verified at the various clinic visits by objective measures, such as exhaled carbon monoxide or cotinine assays, potentially resulting in misclassification with respect to smoking category. However, since UPLIFT was not a smoking cessation study, it is unlikely that subjects would purposefully mislead the investigators as to their true smoking status. Fourthly, the analyses reported herein are based on a post hoc stratification of the subjects into smoking categories based on their smoking behaviour not only at study entry but also during the course of the trial. Consequently, the validity of the p-values for assessing effects of treatment are not completely supported by randomisation arguments, particularly given the imbalance between treatment groups within levels of post hoc stratification that was observed at baseline. Instead of the stronger causality conclusions that could reasonably be inferred, the use of post-randomisation defined subgroups implies that observed differences between randomisation groups are associated with (and not necessarily caused by) differences in treatments. Finally, premature discontinuation was more likely to occur in those with more severe disease and

TABLE 6 Hazard ratios and associated 95\% confidence intervals for tiotropium to placebo for all-cause mortality according to smoking behaviour according to treatment group

\begin{tabular}{|c|c|c|c|c|}
\hline & Subjects $n$ & Mortality rate $\%$ & $\begin{array}{c}\text { Hazard ratio } \\
\text { tiotropium/control }\end{array}$ & $95 \% \mathrm{Cl}$ \\
\hline \multicolumn{5}{|l|}{ On treatment } \\
\hline Continuing smokers & 846 & 16.4 & 1.2 & $0.85-1.69$ \\
\hline Intermittent smokers & 1545 & 10.0 & 0.87 & $0.63-1.19$ \\
\hline Continuing ex-smokers & 3534 & 13.3 & 0.79 & $0.66-0.95$ \\
\hline Intermittent smokers & 1545 & 11.2 & 0.85 & $0.63-1.14$ \\
\hline Continuing ex-smokers & 3534 & 16.2 & 0.81 & $0.69-0.96$ \\
\hline \multicolumn{5}{|c|}{ Vital status at 4 yrs +30 days } \\
\hline Continuing smokers & 846 & 18.8 & 1.24 & $0.90-1.70$ \\
\hline Intermittent smokers & 1545 & 11.8 & 0.90 & $0.67-1.20$ \\
\hline
\end{tabular}


preferentially occurred in the placebo group [3]. As a result, a healthy survivor effect occurs more frequently in the placebo group, which would bias the results against the active drug, tiotropium, and suggests that the actual efficacy may be greater than that observed. The statistical approaches attempting to account for this problem are unlikely to fully adjust for the bias.

In summary, a subgroup analysis by smoking status was performed in 5,925 UPLIFT participants with available trial data who were classified as continuing smokers, intermittent smokers or continuing ex-smokers on the basis of their smoking behaviour at both baseline and during the course of the trial. Continuing and intermittent smokers showed worse outcomes in terms of lung function decline than continuing exsmokers and tiotropium had no discernible association with lung function decline in any smoking subgroup. Conversely, tiotropium was associated with significant long-term benefits compared with placebo with respect to improvements in preand post-bronchodilator lung function irrespective of smoking status, reductions in the risk for and frequency of exacerbations across all smoking categories that was significant in the continuing ex-smokers, and statistically significant improvements in health-related quality of life in both continuing smokers and continuing ex-smokers. Tiotropium also was associated with a significant reduction in all-cause mortality in the continuing ex-smokers with a trend toward a mortality benefit in the intermittent smokers but not the continuing smokers. These observations should be considered in the setting of the trial in which patients were permitted to use other respiratory medications as prescribed by their physicians, except for inhaled anticholinergics, during the trial. These findings indicate that long-term treatment with tiotropium is associated with a beneficial impact on lung function and patient-reported outcomes across different smoking behaviours, although differences in the magnitude of benefit may occur.

\section{CLINICAL TRIALS}

This trial has been registered with www.clinicaltrials.gov (NCT 00144339).

\section{STATEMENT OF INTEREST}

Statements of interest for all the authors and for the study itself can be found at www.erj.ersjournals.com/misc/statements.dtl

\section{REFERENCES}

1 Moita J, Barbara C, Cardoso J, et al. Tiotropium improves FEV1 in patients with COPD irrespective of smoking status. Pulm Pharmacol Ther 2008; 21: 146-151.

2 Rennard SI, Serby CW, Ghafouri M, et al. Extended therapy with ipratropium is associated with improved lung function in patients with COPD. Chest 1996; 110: 62-70.

3 Tashkin DP, Celli B, Senn S, et al. A four-year trial of tiotropium in chronic obstructive pulmonary disease. $N$ Engl J Med 2008; 359: 1543-1554.

4 Decramer M, Celli B, Tashkin DP, et al. Clinical trial design considerations in assessing long-term functional impacts of tiotropium in COPD: The Uplift Trial. COPD: J Chronic Obstruct Pulmon Dis 2004; 1: 303-312.

5 Barnes PF. Corticosteroid resistance in airway disease. Proc Am Thorac Soc 2004; 1: 264-268.

6 Braganza G, Chaudhuri R, Thomson NC. Treating patients with respiratory disease who smoke. Ther Adv Respir Dis 2008; 2: 95-107.

7 Barnes PJ, Ito K, Adcock IM. Corticosteroid resistance in chronic obstructive pulmonary disease: inactivation of histone deacetylase. Lancet 2004; 363: 731-733.

8 van Overveld FJ, Demkow U, Gorecka D, et al. Differences in responses upon corticosteroid therapy between smoking and nonsmoking patients with COPD. J Physiol Pharmacol 2006; 57: Suppl. 4, 273-282.

9 Chalmers GW, Macleod KF, Little SA, et al. Influence of cigarette smoking on inhaled corticosteroid resistance in asthma. Thorax 2002; 57: 226-230.

10 Lazarus SC, Chinchilli VM, Rolling NJ, et al. Smoking affects response to inhaled corticosteroids or leukotriene receptor antagonists in asthma. Am J Respir Crit Care Med 2007; 175: 783-790.

11 Livingston E, Thomson N, Chalmers G. Impact of smoking on asthma therapy: a critical review of clinical evidence. Drugs 2005; 65: 1521-1536.

12 Bukowskyj A, Nakatsu K, Munt P. Theophylline resassessed. Ann Intern Med 1984; 101: 63-73.

13 Anthonisen NR, Connett JE, Kiley JP, et al. Effects of smoking intervention and the use of an anticholinergic bronchodilator on the rate of decline in FEV1. The Lung Health Study. JAMA 1994; 272: 1497-1505.

14 Scanlon PD, Connett JE, Waller L, et al. Smoking cessation and lung function in mild-to-moderate chronic obstructive pulmonary disease: the Lung Health Study. Am J Respir Crit Care Med 2000; 161: 381-390.

15 Anthonisen NR, Skeans MA, Wise RA, et al. The effects of a smoking cessation intervention on 14.5-year mortality. Ann Intern Med 2005; 142: 233-239. 